\title{
MAGNETIC FIELDS AND BLACK HOLES IN GALACTIC NUCLEI
}

\author{
R. D. BLANDFORD
}

130-33 Caltech, Pasadena, CA 91125, USA

\section{Introduction}

Recent observations have strengthened the dynamical arguments for the presence of black holes in X-ray binary systems, the Galactic center and the nuclei of the majority of bright galaxies. They have also drawn attention to the fact that accretion does not always proceed in the simple, stationary manner envisaged in early theories. I wish to discuss four related topics that are relevant to understanding the behavior of accreting black holes. (In view of the length constraints imposed by conference proceedings I shall make no attempt to give adequate bibliographic references for what follows.)

\section{Accretion Disk Magnetic Field}

It now appears to be fairly certain that magnetic fields are responsible for the torque in accretion disks. (For an excellent review, see Balbus \& Hawley 1997). Unmagnetized Keplerian disks are stably stratified. However, when a small, vertical magnetic field is introduced, the slow magnetosonic mode is destabilized by the rotation. The magnetic tension restoring force is overcome by centrifugal force, in analogy to what happens when two orbiting satellites are connected by an elastic rope. A gas element at radius $r+\delta r$ will fall behind a second element at radius $r$ to which it is connected by magnetic field. The magnetic stress will exert a positive torque on the outer element, increasing its angular momentum $\ell$. However, for circular orbits, $r \propto \ell^{2}$. Therefore the radius of the outer element will increase with time. The instability grows on a dynamical timescale $\sim \Omega^{-1}$ for lengthscales $\sim a / \Omega$, where $a=(4 \pi \rho)^{-1 / 2} B$ is the Alfvén speed. The instability is more powerful than 
conventional dynamo modes. In the non-linear phase, alternating layers of reversed field are created in the disk which becomes Kelvin-Helmholtz, (and presumably tearing-mode), unstable. The net result is that the shear stress in the disk is dominated by Maxwell (ie magnetic) not Reynolds (ie fluid) stress. If we introduce a simple $\alpha$ parametrisation, then the internal shear stress can be written $\alpha P$, where $P$ is the pressure and $\alpha \sim 0.01$ (Hawley et al. 1995, Brandenburg et al. 1995). However, magnetoturbulence is a property of the flow and the flux and not simply the fluid. This means that the details of the non-linear saturation depends upon the boundary conditions. Numerical simulations by Hawley et al(1995) indicate that when there is a fairly strong uniform vertical field $B_{0}$ threading the disk, with $\beta_{0}=8 \pi P / B_{0}^{2}<\alpha^{-2}$, the effective value of $\alpha$ rises to $\beta_{0}^{-1 / 2}$.

Now let us consider a stratified disk with dense cool gas in the midplane, sandwiched between warmer more tenuous gas and terminating in a hot corona. If we adopt the above prescription to calculate the total torque, we find that it is given by

$$
G=4 \pi r^{2} \int_{0}^{z_{\text {crit }}} d z \max \left[\alpha P, B_{0}(P / 4 \pi)^{1 / 2}\right]
$$

The upper limit, $z_{\text {crit }}$ is given approximately by the requirement that the Balbus-Hawley instability still be able to grow. This is located at the height where $\beta_{0} \sim 1$. It is clear from the form of this integral, that it is quite possible for most of the inflow and the energy release to occur away from the midplane where $z \sim z_{\text {crit }}$ and for most of the dissipation to happen in the corona.

This then raises the question of whether an external magnetic torque, associated with the vertical field $B_{0}$ can be competitive with the internal torque. The magnetic shear stress applied to the disk surface is $B_{z} B_{\phi} / 4 \pi$. If we adopt the above prescription for the internal torque and suppose that it dominates the angular momentum transport, then the ratio of the two torques can be written in the form

$$
\frac{G_{\text {ext }}}{G \text { int }} \sim \frac{B_{\phi} r}{B_{0} z_{\text {crit }}} \sim\left(\frac{\dot{M}_{W}}{\dot{M}}\right)\left(\frac{r_{A}}{r}\right)^{2}\left(\frac{r}{z_{\text {crit }}}\right)
$$

where $\dot{M}_{W}$ is the wind discharge and $r_{A}$ is the radius of the Alfvén critical point for a field line with foot point radius $r$. Typically $r_{A} / r \sim 10$ and there is no good reason to suppose that this ratio is small. Indeed it may even exceed unity so that most of the angular momentum liberated by the inflowing gas over some intervals of radius is carried away by winds or jets. This, in turn, would also imply that the power carried away as jet kinetic energy exceeds that released within the disk. 
Another unanswered question concerns the energy release associated with the internal torque. A magnetic torque $G$ acting within an accretion disk, does work on the exterior disk at a rate $G \Omega$. This corresponds to an outwardly directed flux of angular momentum. In addition, there is an inevitable release of binding energy to the gas of rate per unit radius $-G d \Omega) / d r$. (This term is the source of the factor three that relates the true local energy release to the rate of release of gravitational binding energy in a standard disk model.) If the torque is regarded as frictional or viscous in character, then this can be regarded as a true dissipation with an entropy increase and the production of heat, which may or may not be radiated away. There are two ways to bring about this dissipation, magnetic reconnection and driving a turbulent cascade. In magnetic reconnection, an "anomalous", ie enhanced resistivity develops around a neutral point and as gas flows through this region, its entropy increases though Ohmic heating. In the case of a cascade, a purely hydromagnetic development of the Balbus-Hawley instability may create disturbance on some "outer" scale that is far too large for microscopic dissipation to operate, though still small compared with the thickness of the disk. If the energy deposited at this scale forms a hydromagnetic turbulence spectrum extending down to small length scales on which ion-cyclotron or transit time damping can operate, then we can regard the dissipation as local (cf Grusinov 1997, preprint, Quataert 1997 preprint). Alternatively, the torque need not be dissipative. Loops of magnetic flux may be created on scales that are sufficiently large compared with the local scale height that they can rise buoyantly in the effective gravitational field of the disk and escape into the corona via Parker and allied instabilities, without local dissipation. (Indeed if the magnetic loops are formed on too large a scale, it may no longer be permissible to treat the torque as local.) In summary, if global disk evolution is to be encapsulated in the $\alpha$ prescription, it may be necessary to distinguish two values of this parameter, one defined, as usual, by the shear stress and the second by the entropy production. Both of these quantities could be measured in numerical simulations of sufficient scale that allow magnetic flux to escape.

The issue of magnetic dissipation is particularly important for advectiondominated accretion flows, "ADAFs" (eg Narayan \& Yi 1995). The observational stimulus for re-considering these solutions is the low apparent radiative efficiency of some accreting black holes. A good example is the Galactic center where it is argued that the mass supply rate is $\sim 10^{22} \mathrm{~g}$ $\mathrm{s}^{-1}$ (Falcke \& Melia 1997), while the bolometric luminosity does not exceed $\sim 10^{36} \mathrm{erg} \mathrm{s}^{-1}$. The radiative efficiency is then $\epsilon \sim 10^{14} \mathrm{erg} \mathrm{g}^{-1} \equiv 10^{-7} c^{2}$, not much better than gunpowder and hardly an advertisement for "gravity power"! Even though both these numbers are contestable, $\epsilon$ must be 
orders of magnitude less than the value $\epsilon \sim 10^{20} \mathrm{erg} \mathrm{g}^{-1}$ usually assumed. ADAFs address this problem by making two assumptions. The first is that the torque and dissipation are both described by a common value of $\alpha$ which is large enough so that the gas remains relatively tenuous. The second assumption is that the associated dissipation just heats the ions not the electrons. The electrons maintain a Maxwellian distribution with temperature much less than that of the ions through Coulomb scattering and synchrotron self-absorption. For sufficiently low mass accretion rate, $\dot{M}<\dot{M}_{c r i t} \equiv 10^{24} \alpha^{2}\left(M / 10^{6} \mathrm{M}_{\odot}\right) \mathrm{g} \mathrm{s}^{-1}$, the disk will thicken within some transition radius and maintain a quasi-spherical inflow of low radiative efficiency, a feature which distinguishes black hole accretion from accretion onto neutron stars. It has proved possible to model the observed spectra of several black holes sources in this manner. Now, it is crucial that the electrons not be accelerated to suprathermal energies where they can become very efficient radiators through the synchrotron and inverse Compton processes. This is in marked contrast to what is assumed in models of X-ray Seyfert galaxies, where it is supposed that electrons in a hot corona above a cooled accretion disk as is supposed to occur when $\dot{M}>>\dot{M}_{\text {crit }}$, are highly efficient sources of a non-thermal power law, hard X-ray spectrum that illuminates the disk. It is not clear why electrons should behave so differently under apparently similar physical conditions.

\section{Jets}

Just as observations have forced the issue and limited our options in understanding disk accretion, so our knowledge of the relationship between disks and jets has advanced as a result of an impressive suite of observations. We are starting to observe relativistic jets on a very small scale. The best case so far is M87 where it appears from VLBI observations (Junor \& Biretta $1995)$, that the eastern jet is at least partially collimated on a transverse length scale $\sim 3 \times 10^{16} \mathrm{~cm} \sim 60 \mathrm{~m}$. In addition, observations of the jet in the LINER galaxy NGC 4258 have verified that the flat spectrum radio core is really the synchrotron self-absorbed inner region of the approaching jet instead of, for example, a spherical isotropic halo surrounding the central black hole. Indeed, in this source, the absence of radio emission from the dynamical location of the black hole sets an important constraint on the character of the accretion flow. (Herrnstein, private communication.) Generalizing from these special cases and guessing that all black hole jets are made by a common mechanism, we must infer that the power for these jets derives from very close to the black hole, and possibly from the hole itself.

Another important observational development is the association of $\gamma$-ray sources with blazars. Here it appears that $\sim 1 \mathrm{GeV} \gamma$-rays are focused into 
narrow cones with opening angles $\sim 5^{\circ}$, presumably by relativistic motion of a scattering cloud of electrons and positrons. However, these $\gamma$ rays can only be emitted outside the $\gamma$-ray photosphere or gammasphere where the opacity to pair production on the ambient X-rays falls below unity. In some sources, the gammasphere appears to be at $\sim 1000 \mathrm{~m}$ at least. The $\gamma$-rays are presumably created by electrons and positrons through inverse Compton scattering. However, these electrons and positrons cannot, themselves be the agency though which power is transported from the vicinity of the hole to the emission region because they are subject to a strong radiative drag. The only two viable means of carrying jet power all the way from $r \sim m$ to the emission region appear to be using protons and electromagnetic field (ie Poynting flux). Now I am not aware of any viable mechanism for collimating protons and accelerating them quickly to ultarelativistic speed that does not involve strong electromagnetic field and for this reason, it seems more reasonable to suppose that the jet power is intially of electromagnetic. In this case, the principal function of the plasma, at least close to the hole is to supply currents and charge density. Electromagnetic energy extraction is quite familiar. For example, the rotational energy, that is extracted from the neutron star in the Crab Nebula, is transported initially as a Poynting flux and then generally supposed to be transformed into a particle flux in the outflowing relativistic wind. Although the details may be different, the same process may be at work in relativistic jets.

It is generally supposed that jets are launched perpendicular to the plane defined by a thin accretion disk. It is also commonly believed that this coincides with the equatorial plane of a Kerr hole, into which matter settles under a combination of precessional and frictional torques. This is not, of course, the only possibility. For example, if there is an ADAF, this may include a funnel within which gas can be given the escape velocity by adding a small amount of entropy. (However, it is not clear that this is sufficient to explain the ultrarelativistic speeds that are observed.) There is also the associated problem of confining the jet outflow. Again attention has centered around magnetic fields under most circumstances, although it is not clear if the principal agency is the toroidal field exerting a hoop stress or the poloidal field exerting a magnetic pressure gradient. In addition, the stability of hydromagnetically collimated jet outflows is problematic. In one attractive, hybrid model the jet is collimated by toroidal magnetic field that is spun off an orbiting accretion disk. An axisymmetric field is probably unstable and may well evolve into a precessing jet solution with finite amplitude, rather like the deformation of a buckled strut. This would actually account quite well for the kinematic behavior of compact radio sources as observed by VLBI and perhaps even the observed precession in SS433 and GRO J 1655-40. It also suggests a mechanism for converting 
electromagnetic energy into particle kinetic energy. As the jet propagates away from the hole, the magnetic field will become increasingly toroidal and perhaps subject to tearing mode instability which can reconnect the field so that the magnetic energy declines roughly with radius as $\propto r^{-4}$. This allows the jet outflow to become particle-dominated at some distance from the black hole and provides a mechanism for accelerating electrons and positrons in situ to $\sim \mathrm{GeV}$ energies near the gammasphere.

In this interpretation, the emergent jet would have two components, an ultrarelativistic core and a more slowly moving sheath derived from the accretion disk over several decades of radius. The sheath is supposed to be responsible for collimating the jet core, although it may not be too well collimated itself. It is possible that the eventual jet speed depends upon the relative thrusts of the core and the jet. When the core carries a relatively hight thrust (and power), it will eventually accelerate the sheath to its speed. Conversely when the core power is low, it will be decelerated by the sheath to a low speed which may eventually be not much faster than the local escape velocity. This distinction may be all that is necessary to explain the differences between FR1 and FR2 sources. Many of our uncertainties concerning the behavior of jets will be resolved by performing careful three dimensional MHD simulations which more often than not produce surprising results (eg Meier et al 1997).

\section{Energy Extraction from Black Holes}

As hinted above, and discussed extensively elsewhere, it seems quite reasonable that jet cores are powered by Poynting flux extracted directly from the spin of the central black hole. For example fields of strength $\sim 10^{4} \mathrm{G}$ threading $\mathrm{a} \sim 10^{9} \mathrm{M}_{\odot}$, hole are adequate to account for the most energetic radio galaxies.

However this is not the only way to extract energy from the hole. Another possibility is that magnetic flux is convected radially inward by the accreting gas until it threads the event horizon. The field lines will be frozen into the inner accretion disk and move with its angular velocity. (This is like the "propellor" mechanism that takes place when there is accretion onto a rapidly spinning neutron star except that now the field lines are attached to the disk.) Now, as long as the hole's angular velocity exceeds that of the disk gas (and this will be true for Keplerian orbits beyond the marginally stable orbit if $\Omega_{H}>0.093 \mathrm{c} / \mathrm{m}$ ), there will be a positive, magnetic torque acting on the disk increasing its angular momentum at the expense of the spin of the hole. There will be negligible energy dissipation at the disk; this will take place within the event horizon, increasing the hole's irreducible mass. Nonetheless, there will energy production, as explained above, associated 
with the radial transport of angular momentum.

Let us suppose that the hole angular velocity approaches its maximal value and the vertical field, threading both the disk and the hole has a strength $B_{0}$ close to the maximum value that it can still have without disrupting the disk. It is then straightforward to show that the ratio of the torque that the hole exerts on the disk $G_{0}$ to the flux of angular momentum carried by the inflowing matter is roughly

$$
\frac{G_{0}}{F_{\ell}} \sim \frac{\ell}{z_{\text {crit }} v_{r}}
$$

where $\ell$ is the specific angular momentum of the inner disk and $v_{r}$ is the radial velocity. In a steady state, the rate of energy release per unit radius is

$$
D(r)=\frac{3 \ell\left(G_{0}+\dot{M} \ell(r)\right)}{2 r^{3}}
$$

It is clearly possible for the hole to be a powerful source of angular momentum for the inner disk that can arrest the accretion and drive a lot of the gas away. Indeed, it is possible to imagine torques exerted by giant coronal arches carrying this torque outward through many decades of radius with little dissipation and removing most of the gas supplied at large radius in the form of a wind. This may provide an alternative explanation to ADAFs for tiny radiative efficiency. In addition, it seems quite likely that if the hole can attenuate the accretion as suggested here, it will do so in a timedependent manner. This could have relevance to the limit cycle oscillations observed in Galactic black hole binaries. These possibilities deserve more attention.

\section{White Dwarf Capture}

I will conclude with a slightly different application of these ideas that was originally stimulated by observations of $\gamma$-ray bursts (Blandford \& Ostriker 1997 , in preparation). The question is "What happens when a massive black hole captures a white dwarf?". Now it is easy to show that holes with masses as large as those thought to reside in the nuclei of galaxies like our own, $\left(M>10^{6} \mathrm{M}_{\odot}\right)$ can and will swallow white dwarfs whole without any conspicuous display. However, most galaxies are dwarf galaxies and it is a reasonable hypothesis that they contain lower mass black holes with $M \sim 10^{5} \mathrm{M}_{\odot}$. Under these circumstances, a white dwarf with a small enough impact parameter can be captured directly into a relativistic orbit though a combination of gravitational bremsstrahlung and tidal excitation of oscillations. It appears that this capture is likely to occur at an interesting rate, $\sim 1 \mathrm{Myr}^{-1}$ per galaxy. 
What happens next is quite interesting. Provided that there are not too many distant encounters near apbothon, the orbit will gradually circularize largely through the gravitational radiation. It will probably also settle into the equatorial plane of the hole assuming that it is spinning. (It turns out that this evolution is only possible for prograde captures.) The orbit will gradually shrink until it reaches its Roche radius $r_{R} \sim 3-5 \mathrm{~m}$ at which point it will pour through its $L_{1}$ point to form an orbiting, degenerate ring. (This happens on a dynamical time scale because the natural radius of a white dwarf increases with decreasing mass, whereas the radius of the Roche lobe decreases.) Now this ring will be subject to two type of instability. Firstly, there will be "Papaloizou-Pringle" instabilities which may destroy the ring soon after it forms. However, secondly there are Balbus-Hawley type instabilities that will amplify magnetic field and may create loops which escape under buoyancy forces and can link the torus. If the second instability can grow before the first, then it is possible to build up the field to a strength as large as $\sim 10^{11-12}$ G. Now much of this magnetic flux will cross the event $h$ orizon of the hole and can extract its spin energy. It is not clear at this stage if all of this can occur fast enough to account for a $\gamma$-ray burst, but it does seem likely that events like this ought to occur if the nuclei of dwarf galaxies contain $\sim 10^{5} \mathrm{M}_{\odot}$ black holes and should be observable. As most of the steps in this evolution depend upon secure physics, like the equation of state for degenerate matter, it should be possible to simulate it with some confidence, and perhaps ultimately use it to provide a strong field test of general relativity.

\section{Acknowledgements}

I thank Jerry Ostriker for patient collaboration on the contents of section 5 . Andrei Gruzinov, John Hawley, Rob Nelson and Ramesh Narayan are also thanked for helpful conversations. Support under NSF grant AST 95-29170 is gratefully acknowledged.

\section{References}

Balbus, S. A. \& Hawley, J. F. 1997 RMP (in press)

Brandenburg, A., Nordlund, Å., Stein, R. F. \& Torkelson, U. 1995 ApJ 446741

Falcke, H. \& Melia, F. 1997 ApJ 479740

Hawley, J. F., Gammie, C. F. \& Balbus, S. A. 1995 ApJ 440742

Junor, W. \& Biretta, J. A. 1995 AJ 109500

Meier, D. L., Edgington, S., Godon, P., Payne, D. G. \& Lind, K. R. 1997 Nature 388350 Narayan, R. \& Yi, I. 1995 ApJ 45270 